Journal for ImmunoTherapy of Cancer Weber J, et al. Immunotherapy to treat malignancy in patients with pre-existing autoimmunity. Journal for ImmunoTherapy of Cancer 2020;8:e000356. doi:10.1136/jitc-2019-000356

Accepted 21 March 2020

\title{
Immunotherapy to treat malignancy in patients with pre-existing autoimmunity
}

\author{
Patrick Boland, ${ }^{1}$ Anna C Pavlick, ${ }^{1}$ Jeffrey Weber, ${ }^{1}$ Sabina Sandigursky ${ }^{1,2}$
}

\begin{abstract}
In the past 10 years, immune checkpoint inhibitors (ICls) have become an additional pillar of cancer therapy by activating the immune system to treat a number of different malignancies. Many patients receiving ICls develop immune-related adverse events (irAEs) that mimic some features of classical autoimmune diseases. Unfortunately, patients with underlying autoimmune conditions, many of whom have an increased risk for malignancy, have been excluded from clinical trials of ICls due to a concern that they will have an increased risk of irAEs. Retrospective data from patients with autoimmune diseases and concomitant malignancy treated with ICls are encouraging and suggest that ICls may be tolerated safely in patients with specific autoimmune diseases, but there are no prospective data to guide management. In this manuscript, we review the relationship between preexisting autoimmune disease and irAEs from checkpoint inhibitors. In addition, we assess the likelihood of autoimmune disease exacerbations in patients with preexisting autoimmunity receiving $\mathrm{ICl}$.
\end{abstract}

\section{CANCER AND AUTOIMMUNE DISEASE}

Immune surveillance against cancer can fail due to suppression of antitumor immunity or immune editing, which lead to persistence and proliferation of tumor cells. In contrast, autoimmunity arises from an aberrant immune response to self-antigens, either directly due to recognition of these antigens as non-self or indirectly through disruptions in the microbiome, for example. Patients with chronic autoimmune illnesses are predisposed to lymphoproliferative conditions and solid malignancies either due to chronic inflammation or treatment-related immunesuppressive effects.

Patients with rheumatoid arthritis and lupus have high rates of malignant lymphoma and non-small-cell lung cancer (NSCLC). ${ }^{1}{ }^{2}$ In one study of patients with newly diagnosed metastatic melanoma, $28 \%$ had a coexisting autoimmune disease (AID) at the time of cancer diagnosis. ${ }^{3}$ A similar study of NSCLC found that nearly 16000 patients with stage III/IV NSCLC of 129000 diagnosed every year had a comorbid AID. ${ }^{4}$

Immune checkpoint inhibitors (ICIs) interfere with coinhibitory $\mathrm{T}$ cell mediators such as cytotoxic $\mathrm{T}$ cell lymphocyte-associated protein 4 (CTLA-4) and programmed cell death-1 (PD-1). Monoclonal antibodies blocking the CTLA-4 and PD-1 pathways convert functionally exhausted $\mathrm{T}$ cells into activated, cytotoxic cells capable of clinically important antitumor effects. These drugs have revolutionized the field of oncology by leading to durable responses in many cancer types.

Clinical trials that led to United States Food and Drug Administration (FDA) approval of anti-CTLA-4 and anti-PD-1 agents have shown that their toxicity is due to immune-mediated inflammatory changes in healthy tissues. The concern that patients with pre-existing AIDs would experience disease exacerbation or increased autoimmune toxicity from ICI resulted in those patients being excluded from the clinical trials that led to the registration of ICIs.

Data from studies of patients with concomitant diagnoses of cancer and autoimmunity suggest that tens of thousands of patients might be excluded from treatment with ICIs even if they might provide clinical benefit due to a concern for exacerbation of an autoimmune condition. ${ }^{5}$ This led to a re-examination of the omission of patients with pre-existing autoimmunity from trials of ICIs, and to reassess guidelines for ICI treatment that recommend that those patients not be treated with ICI. In 2020, AID is not an absolute contraindication for the use of ICIs; however, many oncologists are still wary of using ICIs in this patient population as the exact risks remain unclear. In this review, we will explore shared mechanisms of immunerelated adverse events (irAEs) and AIDs, the prognostic significance of serum autoantibodies, and evidence for the safety and efficacy of ICIs in patients with pre-existing autoimmunity.

\section{Immune-related adverse events}

Ipilimumab (IPI), a fully human anti-CTLA-4 antibody, was the first FDA-approved immunotherapy to show a survival advantage in 
metastatic melanoma in a phase III trial. However, it was also associated with a high percentage of irAEs of any grade affecting approximately $60 \%$ of patients. ${ }^{6}$ Further, $10 \%-30 \%$ of the immune-related toxicities with IPI were considered severe and potentially life threatening (grade 3/4) based on the National Cancer Institute's (NCI) Common Terminology Criteria for Adverse Events. In addition, there is a dose-dependent effect of IPI on toxicity, with higher rates observed in the $10 \mathrm{mg}$ / $\mathrm{kg}$ groups compared with $3 \mathrm{mg} / \mathrm{kg}$. ${ }^{7}$ The most frequently occurring grade $3 / 4$ irAEs in patients treated with IPI are diarrhea/colitis in approximately $20 \%$ of patients. Grade 3/4 colitis often occurs within 8-12 weeks of treatment initiation. Other toxicities commonly seen in IPI-treated patients include pruritus, hepatitis and endocrine dysfunction. Skin and gastrointestinal toxicity occur early after IPI treatment, with liver toxicity often seen later in the course of treatment usually within 4-9 weeks of starting treatment compared with endocrine toxicities, which can develop later, after $7-10$ weeks. ${ }^{8}$ Recent preclinical data suggest that direct delivery of the antiCTLA-4 antibody can uncouple the antitumor effect from autoimmune toxicity. ${ }^{9}$

A characteristic toxicity of ICIs is hypophysitis. Prior to the use of IPI hypophysitis was regarded as exceedingly rare. Currently, it is important to screen for hypophysitis in patients receiving ICI as significant morbidity may ensue if the diagnosis is delayed.

Nivolumab, pembrolizumab and cemiplimab, all monoclonal antibodies blocking the PD-1 receptor, have similar toxicities. Anti-PD-1 therapeutics are less toxic than IPI, with approximately $50 \%-70 \%$ of patients developing irAEs of any grade and approximately $10 \%-15 \%$ developing grade $3 / 4$ toxicity. ${ }^{10-12}$ The most common toxicities of any grade include fatigue, rash, pruritus, arthralgia, diarrhea/colitis, hepatitis and endocrinopathies. Generally, the majority of irAEs in patients on anti-PD-1 therapy occur within the first 6 months of treatment. ${ }^{13}$

Thus far, three agents targeting PD-L1 have been FDAapproved, atezolizumab, durvalumab and avelumab. Interestingly, anti-PD-L1 agents appear to have less pulmonary toxicity compared with anti-PD- 1 agents $(4.9 \%$ vs $1.9 \%) .{ }^{14}$ Perhaps, this is due to PD-L2 (the second known PD-1 ligand) signaling remaining unaffected, allowing for partially preserved PD-1 mediated tolerance. These findings were also observed in a meta-analysis including $>5000$ patients with NSCLC..$^{15}$ Avelumab-related infusion reactions have been reported in approximately $25 \%$ of patients ${ }^{16}$ likely owing to the fully humanized Fc tail of the antibody. However, no studies have directly compared anti-PD-1 and anti-PD-L1 antibodies in the same population, suggesting that some differences in toxicity profile may due to the particular treatment indication.

The combination of IPI with nivolumab has resulted in increased benefit compared with either agent alone in metastatic melanoma and renal cell carcinoma and has led to FDA approval of combination therapy in those tumor types, but at the cost of increased incidence and severity of irAEs. ${ }^{17-22}$ In addition, irAEs emerged earlier after combination therapy than with single agent IPI or nivolumab. Trials using combination therapy for the treatment of melanoma targeting the PD-1 and CTLA-4 pathways have shown a marked increase of grade III/IV irAE rates in the Checkmate 069 trial which compared combination to IPI monotherapy (54\% vs $24 \%)$ and Checkmate 067 which compared combination therapy to IPI alone or nivolumab monotherapy. This trial also revealed enhanced toxicity for the combination versus either therapy alone with grade III/IV irAEs ( $59 \%$ vs $28 \%$ vs $21 \%$, respectively). ${ }^{18} 21$

Of all the organ systems involved with irAEs, cardiac toxicity carries the greatest mortality risk. Myocarditis is more likely to occur in patients receiving combination checkpoint blockade and is more likely to be fatal in those patients. ${ }^{23}$ Zamami et al recently published factors associated with risk for myocarditis. They identified that female patients and patients over the age of 75 were at greatest risk for myocarditis. ${ }^{24}$ In a review of WHO database of immunotherapy-associated myocarditis, mortality was reported to be $67 \%$ for patients who received combination therapy and $36 \%$ with antiPD-(L) 1 targeting antibodies. ${ }^{25}$ The current hypotheses regarding the development of cardiac toxicity relate to $\mathrm{T}$ cell-mediated infiltration of cardiac tissue in response to cross-reactive antigens. A postmortem analysis from one patient revealed shared clonality of $\mathrm{T}$ cell receptors (TCR) in tumor-infiltrating $\mathrm{T}$ cells and cardiac-infiltrating $\mathrm{T}$ cells without evidence of antibody involvement. $^{23}$

Overall, irAEs are a heterogeneous group of immunemediated phenomena affecting multiple organ systems. Surprisingly, animal models testing checkpoint inhibitors showed adequate anticancer benefit, without evidence of toxicity. Only after their introduction into clinical trials did irAEs become evident and it became clear that reverse translational animal models are desperately needed.

A study looking at efficacy of anti-PD-1 therapy in combination with anti-tumor necrosis factor (TNF) therapy to enhance antitumor efficacy was published suggesting that this combination could achieve a synergistic effect. ${ }^{26}$ More recently, a manuscript highlighted the possibility of uncoupling irAEs from ICI treatment effects in a mouse model. ${ }^{27}$ In their study, the authors treated mice with dextran sodium sulfate (DSS) to chemically induce colitis in mice with tumor. Further, mice were given with combination immunotherapy (anti-PD-1 and anti-CTLA-4) as well as anti-TNF alpha antibody. The data suggested that tumor shrank and colitis improved. The authors concluded that this may be an effective strategy to uncouple toxicity from efficacy. While this study is interesting and can shed some light on basic biology, there are some major caveats. DSS colitis is a chemically mediated disease; it is known to respond well to anti-TNF therapy and animals treated with ICIs do not recapitulate human toxicity. Indeed, better reverse translational models are needed to study irAEs in animals. 
In patients suffering from gastrointestinal irAEs, anti-TNF therapy has been used. In this recent report, five patients were treated with concomitant infliximab and immunotherapy regimen with resolution of gastrointestinal complications and no evidence of malignancy progression. ${ }^{28}$

Data have supported the hypothesis that irAE development is associated with increased progression-free survival (PFS) and overall survival (OS) in a variety of tumor types, especially NSCLC, suggesting shared mechanisms for antitumor effects and irAE development. ${ }^{29-33}$ As our understanding of irAEs with ICIs deepens, we will likely be able to develop more targeted therapies to prevent toxicity while maintaining enhanced antitumor responses.

\section{T cells in irAE pathogenesis}

With the emergence of irAEs, a new field to study their pathogenesis has emerged. To date, the most likely culprit of disease initiation and progression is the T cell. Several translational studies have shed light on the importance of new specific $\mathrm{T}$ cell clones, which can lead to toxicity.

In one manuscript, authors Johnson et al profiled infiltrating immune cells from a patient who developed encephalitis after treatment with pembrolizumab. Interestingly, the areas of inflammation had increased numbers of $\mathrm{T}$ cells with memory phenotypes. In addition, they found three predominant $\mathrm{T}$ cell clones recognizing Epstein-Barr virus (EBV) viral proteins with some matching known HLA-A2-restricted EBV-specific TCRs and cytotoxic CD4+ Tcell clones with high PD-L1 staining. ${ }^{34}$

In a similar study evaluating the etiology of fatal ICIinduced myocarditis, Johnson et al describe clonal infiltrating $\mathrm{T}$ cell populations in common between the tumor and myocardium. ${ }^{23}$ Cardiac histology revealed infiltration of CD3+ T cells and patchy necrosis of myofibrils. The authors hypothesized that there was a shared epitope between the target malignant cells and bystander cardiac myofibrils.

A third study evaluating the pathophysiology of checkpoint-induced pneumonitis (CIP) compared the composition of bronchoalveolar lavage fluid in ICI patients with CIP versus those who were treated with ICI and did not develop CIP. Using multiparameter flow cytometry, the investigators found that bronchoalveolar lymphocytosis could serve as a hallmark for CIP. Specifically, they found increased CD8+ T cells as well as central memory $\mathrm{T}$ cells, decreased numbers of regulatory $\mathrm{T}$ cells and higher numbers of activated macrophages in their bronchoalveolar lavage fluid. ${ }^{35}$

Oh et al hypothesized that IPI would lead to an expansion of tissue-specific $\mathrm{T}$ cell clones, driving irAE development. Instead of an expansion of subclinical tissue-specific clones, they saw an increase in de novo $\mathrm{T}$ cell subpopulations in patients who developed irAEs. This expansion occurred within 2 weeks of treatment, at a time before irAEs usually manifest. Remarkably, the overall diversity of the population (ie, the number of clonal populations detected) induced by IPI predicted which patients exhibited irAEs. Patients who experienced irAEs had greated diversity of $\mathrm{CD} 4+$ and CD8+ $\mathrm{T}$ cells without changes in regulatory $\mathrm{T}$ cells. ${ }^{36}$ These data suggest that anti-CTLA-4 agents might trigger irAEs by induction of a large repertoire of phenotypically distinct $\mathrm{T}$ cell clones, some of which may interact with self-tissue. As some of these distinct $\mathrm{T}$ cells may also have specificity for tumor antigen, it suggests a possible mechanism for improved OS and PFS in patients who develop irAEs, although this remains controversial.

While these studies are incredibly informative, targeting $\mathrm{T}$ cells at large to manage irAEs represents a substantial dilemma since $\mathrm{T}$ cells are also those providing maximal antitumor effect.

\section{Autoantibodies in ICI treatment}

Many AIDs are marked by the presence of autoantibodies. While autoantibodies do not always drive specific AIDs, their presence can often be diagnostic or correlate closely with disease activity. Given the role of immune surveillance in tumor recognition, it was observed that antidoublestranded DNA antibodies and antinuclear antibodies (ANAs) were associated with an improved outcome in patients with colorectal cancer and lung cancer, respectively. ${ }^{37-39}$ Notably, ANAs can lead to opsonization of apoptotic cells and may increase the presentation of autoantigens to immune cells. ${ }^{40} 41$ Thus, from mouse studies, we may hypothesize that in tumors with high cell turnover, autoantibodies might enhance the presentation of cancer antigens to immune cells, improving immune surveillance.

In ICI-treated patients, the connection between autoantibodies and treatment outcome has been evaluated. In a cohort of 137 patients with advanced NSCLC treated with nivolumab or pembrolizumab, patients with classic autoreactive antibodies such as ANAs, rheumatoid factor and antithyroid antibodies had higher PFS (6.5 months, 95\% CI 4.4 to 12.9 ) compared with patients without these autoantibodies (3.5 months, 95\% CI 2.4 to 4.1$).{ }^{42}$ Yet, these autoantibodies were also associated with higher rates of irAEs (OR 3.25, 95\% CI 1.59 to 6.65 , p=0.001) and the irAEs were associated with an increase in PFS and OS. Antithyroid antibodies were correlated with increased rates of ICI-related thyroid dysfunction $(20 \%$ vs $1 \%, \mathrm{p}<0.001)$ and rheumatoid factor was associated with increased rates of cutaneous irAEs ( $47 \%$ vs $24 \%, \mathrm{p}=0.02$ ). Since elevated autoantibody levels and irAEs were shown to correlate in those studies, it suggests that ICIs may induce expansion of subclonal B-cell populations until autoimmune phenotypes emerge.

There is evidence published in recent literature suggesting that subclinical autoantibodies can expand after ICI exposure. In one cohort of five patients treated with BCG and ICI, 1620 autoantibodies were queried in sera both pre-ICI and post-ICI treatment. Three of the patients had minimal expansion of autoantibodies after 
ICI administration (27-71 of the 1620 autoantibodies). The other two had high numbers of autoantibodies in post-treatment sera (505-853 of the 1620 autoantibodies, $\sim 42 \%$ of total). Interestingly, only these two highautoantibody patients developed grade III/IV irAEs, which led to early discontinuation of the trial. Of these two patients, the first had a high number of autoantibodies at baseline, as in a primary AID. The second had a rapid expansion of de novo antibodies after BCG/ICI coadministration. ${ }^{43}$

The autoantibody expansion in ICI-treated patients noted above does not depend on BCG. In a study of 99 seronegative melanoma patients treated with IPI, 19 (19.2\%) developed autoantibodies after anti-CTLA-4 treatment. The development of autoantibodies was positively associated with the development of irAEs, but did not reach statistical significance (OR 2.92; 95\% CI 0.85 to 10.01). However, the development of antithyroid antibodies along with thyroid dysfunction did reach such significance (OR 9.96, 95\% CI 1.94 to 51.1). ${ }^{44}$

Gowen et al evaluated 78 patients with melanoma utilizing high-throughput protein arrays analyzing pretreatment autoantibodies in patients with metastatic melanoma. Their data suggest that measurement of serum autoantibodies from patients without an overt autoimmune clinical phenotype could predict the development and severity of irAEs after ICI administration. ${ }^{45}$

Work evaluating the effect of PD-1 on germinal center $\mathrm{B}$ cells has shown that PD-1 deficiency led to fewer longlived plasma cells due to decreased cytokine production from $\mathrm{T}$ follicular helper cells and germinal center B cell death. However, the remaining plasma cells had a greater affinity for antigen. ${ }^{46}$ Other studies have shown that PD-1/PD-L1 blockade or deletion of PD-1 can lead to an augmented B cell proliferative and antibody response to $\mathrm{T}$ cell-independent antigens as well as enhanced IgG isotype switching and longevity. ${ }^{47}$ These studies suggest a mechanism by which PD-1 blockade could lead to autoantibody expansion and subsequent irAE development.

However, even where autoantibodies seem to play a role in irAE pathogenesis, the mechanism may be slightly different than that seen in traditional AIDs. Stamatouli et al evaluated autoantibodies in patients who developed insulin-dependent diabetes mellitus after ICI therapy. In their study, only $40 \%$ of their patients developed any of the anti-islet autoantibodies traditionally observed in spontaneous type 1 diabetes. ${ }^{49}$ Conversely, in spontaneous type 1 diabetes, these autoantibodies are present in $95 \%$ of patients. ${ }^{50}$

These studies demonstrate that both anti-CTLA- 4 and anti-PD-1/PD-L1 agents can induce expansion of autoantibodies in sera. Further, these autoantibodies often correlate with irAE development and potentially tumor response to ICI. Among other mechanisms, expanded autoantibodies may be responsible for increased autoimmune flares or irAE rates in patients with pre-existing autoimmunity exposed to ICIs.
Safety and efficacy of ICls in patients with pre-existing autoimmune conditions

Recent retrospective data have demonstrated the safety and efficacy of ICIs in patients with pre-existing AIDs. One early case series of two patients with metastatic melanoma and AID treated with IPI (one with multiple sclerosis and one with rheumatoid arthritis) showed that there was no flare of either AID after ICI initiation though both patients were maintained on immunosuppression throughout the 4-cycle treatment course. Of the two patients, one had a marked response to immunotherapy. ${ }^{51}$

Subsequently, a report of seven patients with rheumatoid arthritis and cutaneous melanoma treated with IPI showed that four patients developed a partial response to therapy $(57 \%)$ of which two had sustained responses for greater than 2 years $(29 \%){ }^{52}$ This overall response rate (ORR) $(57 \%)$ exceeds the $11 \%-15 \%$ ORR in the general population. ${ }^{534}$ Including an additional patient with ocular melanoma, five of the patients $(62.5 \%)$ discontinued therapy due to high-grade 3-4 irAEs. While the overall level of irAEs was comparable to that of the general population ( $75 \%$ vs $86.2 \%)$, the rates of more serious high-grade III/IV irAEs were significantly higher in this small cohort $(62.5 \%$ vs $27.3 \%)$, especially grade III/IV colitis ( $50 \%$ vs $14 \%$ ). The largest retrospective study of anti-CTLA-4 in pre-existing autoimmunity included a cohort of 30 patients with a variety of AID, including rheumatoid arthritis, multiple sclerosis, inflammatory bowel disease (ie, ulcerative colitis and Crohn's disease) and systemic lupus erythematosus. Of the 30 patients, $\sim 50 \%$ did not experience disease exacerbation or irAEs after IPI therapy. Additionally, $8(27 \%)$ had flares of their AID requiring treatment with systemic therapy, largely corticosteroids. Grade 3/4 irAEs were observed in $9(33 \%)$ patients, only slightly higher than the rate in the general population $(27.3 \%)$. One patient with underlying psoriasis died due to ICI-related colitis. ${ }^{55}$

Anti-PD-1 antibodies nivolumab and pembrolizumab have been evaluated retrospectively in advanced melanoma in patients with either a pre-existing autoimmune disorder or after the development of high-grade irAEs on IPI. ${ }^{56}$ Of the 52 patients with pre-existing autoimmunity, 20 patients $(38 \%)$ exhibited a flare of their underlying AID requiring immunosuppression. Interestingly, none of the 11 patients with gastrointestinal or neurological AIDs flared. Another 15 patients (29\%) developed an additional irAE, comparable to the rate of irAEs in the general population (21\%) receiving PD-1 antibodies. This suggests that with anti-PD-1 therapy, patients with pre-existing AID may have heightened disease activity, but may not have higher rates of de novo irAEs.

The same authors included data on a cohort of 67 patients who developed irAEs and were treated with immunosuppression after exposure to IPI, then were treated with a PD-1 antibody. ${ }^{56}$ Despite all patients having had an irAE on IPI, only $25(37 \%)$ developed a subsequent irAE on anti-PD-1 therapy. Of these 25, only 2 (3\%) had recurrence of their original irAE. The other 
23 patients developed a new irAE altogether. Additionally, only 14 patients (21\%) developed grade III/IV irAEs. There were no patient deaths.

A German study explored the use of anti-PD-1 in patients with pre-existing AID $(\mathrm{n}=19)$ or IPI-induced irAE $(\mathrm{n}=22) .{ }^{57}$ Of patients with AID, $42 \%$ had a flare of their AID, similar to the $38 \%$ in the aforementioned Menzies study. Sixteen per cent developed a new irAE, which is lower than the 29\% rate of irAEs observed in the prior study. Among the cohort of patients with an IPI-induced irAE, the rates of irAE recurrence on anti-PD-1 were similar to those of the prior study ( $4.5 \%$ vs $3 \%)$.

A number of other retrospective studies have evaluated the use of anti-PD-1/PD-L1 agents in patients with pre-existing autoimmunity. In one cohort of 56 patients with NSCLC and prior AID, $55 \%$ of patients developed an irAE or AID flare. ${ }^{20}$ Among the 21 patients $(38 \%)$ who developed irAEs, 5 (26\%) were grade 3 or 4 . Another study of 45 patients found that $44.4 \%$ of patients developed either an AID flare or irAE. ${ }^{58}$ Lastly, a cohort of 16 patients from the Mayo Clinic had similar rates of irAE or AID flare $(37.5 \%)$, all of which were managed successfully with ICI discontinuation or corticosteroids. ${ }^{59}$

Many of these findings were consolidated in a systematic review that included data from 123 patients drawn from 49 publications. ${ }^{60}$ In that study, $92(75 \%)$ of the patients either had a flare of their pre-existing autoimmunity or developed irAE. There were no differences in the development of adverse events in patients with active versus inactive AID at the time of treatment. However, there was a lower rate of adverse events among those receiving treatment for their AID at time of ICI (59\% vs 83\%). Among the patients who developed irAEs or flares, about $50 \%$ had improvement in their symptoms after discontinuation of ICI. Sixty-two per cent required high-dose corticosteroids and a further $16 \%$ required other immunosuppressive disease-modifying agents (eg, methotrexate, rituximab, infliximab). Only three patients (2.4\%) died of adverse events. In line with prior data linking irAEs with an improved rate of ICI response, $25(50 \%)$ of the patients who developed adverse events (either flare or irAE) had a partial or complete response to therapy, rates at or higher than those in the general population treated with any ICI. Among those without any events, only five $(35.7 \%)$ had a response to therapy. These data suggest that a flare of pre-existing AID, in addition to irAE development, may be associated with tumor response to ICI.

In one study, the use of anti-PD-1/PD-L1 agents was associated with higher rates of existing AID flare $(62 \%$ vs $36 \%$ ) than with IPI, while IPI was associated with more de novo irAEs. Anti-CTLA-4 agents may lead to an increased diversity of $\mathrm{T}$ cell clones, which may explain the development of new irAEs. ${ }^{61}$

The largest retrospective series of patients with AID treated with ICI to date included 112 patients with a variety of cancer types treated with anti-PD-1/PD-L1 and anti-CTLA-4. ${ }^{62}$ In this cohort, $22 \%$ were on immunosuppressive treatment at time of ICI initiation. Of the 112 patients, $47 \%$ had AID flare and $42 \%$ developed de novo irAEs. Only $21 \%$ permanently discontinued ICI and there was one treatment-related death. The $49 \%$ ORR in this population was higher than expected compared with patients without AIDs.

A major limitation of these retrospective studies has been the heterogeneity of AID in the study. Two newer manuscripts have overcome this limitation. In a retrospective evaluation of 102 patients with pre-existing IBD, $41 \%$ had a flare of their IBD compared with $11 \%$ with new IBD in a control group at the institutions. Of those with pre-existing IBD, $21 \%$ had grade 3 or 4 diarrhea. However, no deaths were reported and the response rates for malignancy were comparable to those seen in the relevant clinical trials. ${ }^{63}$

Most recently, a report of 22 patients with rheumatoid arthritis was published by Efuni $e t$ al evaluating risk of flares and irAEs. The authors found that while most patients were clinically quiescent prior to initiating immunotherapy for malignant conditions, at least $50 \%$ of patients flared, which seems to support data from other series. $^{64}$

Overall, these findings suggest that ICIs lead to similar rates of irAEs in patients with pre-existing AIDs compared with those without existing AID and are summarized in table 1.

While these data are rather encouraging, due to the development of AID flares in many of these studies, close monitoring is warranted. Further, it should be noted that many of these analyses include multiple autoimmune disorders, when it is clear they are not all the same. In an ideal world, data should be compared from patients with similar spectra of autoimmune illnesses.

A multidisciplinary team that includes oncologists, rheumatologists and other subspecialties would best serve complex patients with pre-existing autoimmunity and malignancy treated with ICIs. This has already been instituted in major academic centers around the country. ${ }^{65}$ Through this collaborative approach, we may be able to bring much needed treatments to a vulnerable population that could derive significant benefit.

\section{Future directions and conclusion}

Currently approved ICIs act on three molecular targets (CTLA-4, PD-1 and PD-L1) to increase the recognition of tumor cells by $\mathrm{T}$ cells. As an expected consequence of immune activation, irAEs affecting multiple organ systems can develop. While the exact mechanism is unclear, irAEs are thought to result from a highly active immune system and in some cases have been shown to be associated with improved outcomes to ICI therapy. ${ }^{29-32}$

Since ICIs trigger irAEs, patients with pre-existing autoimmunity were initially excluded from receiving ICIs on clinical trials, due to fear of autoimmune exacerbations and increased risk of high-grade irAEs. However, retrospective studies have shown comparable if not greater efficacy and similar side-effect profiles in patients with pre-existing autoimmunity treated with ICI. While most studies did show 


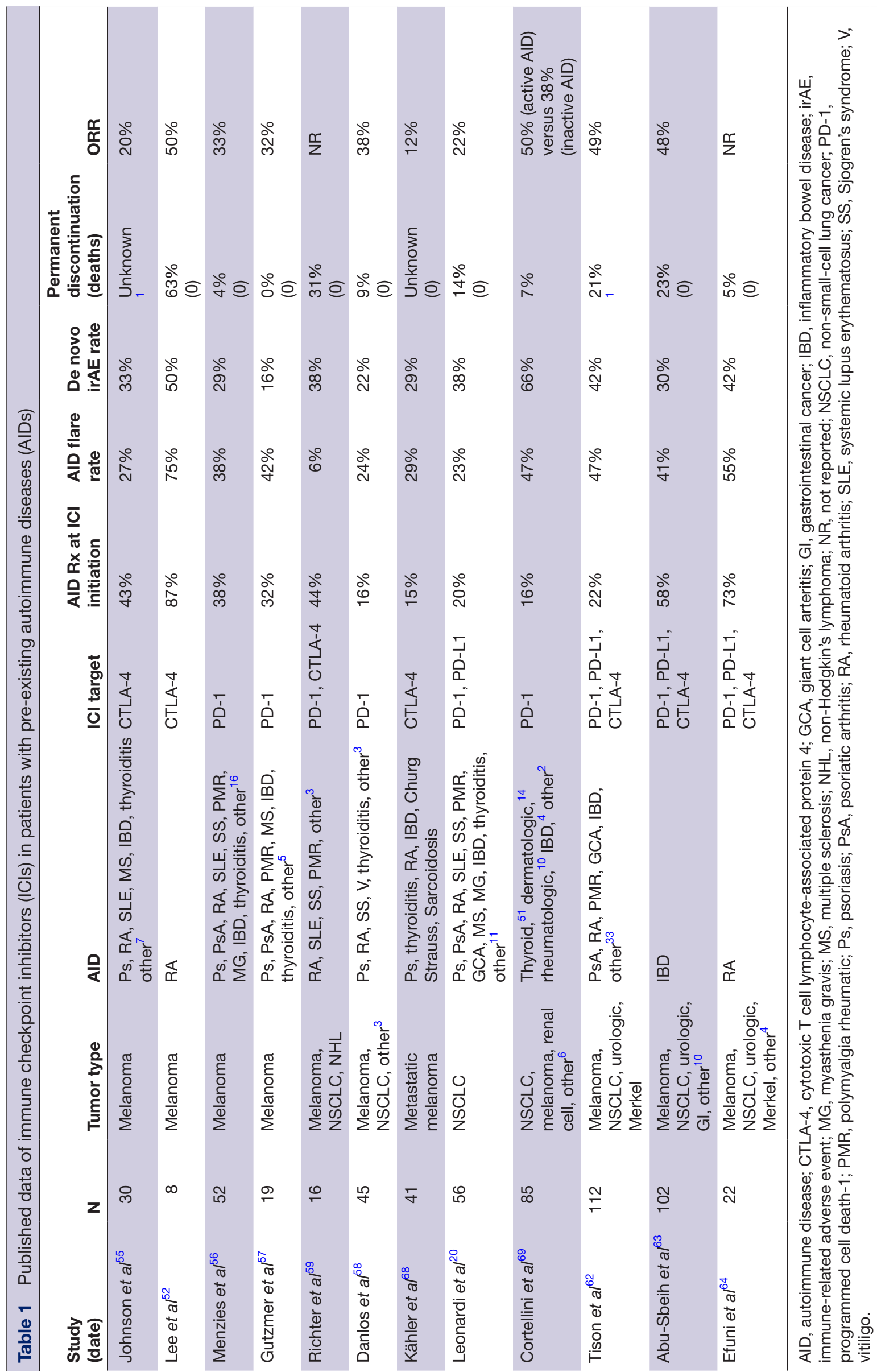


an increased rate of autoimmune flares, these flares were often low grade. In a subset of studies, autoimmune flares correlated with increased response rates to ICI. Currently, documented pre-existing autoimmunity is not a clear absolute contraindication for prescribing immunotherapy, though clinicians in the community may be wary to do so.

It is recommended that oncologists using ICI therapy collaborate closely with specialists well versed in specific autoimmune illnesses. Many centers have formed a network of specialists interested in managing potentially life-threatening toxicity from ICIs. ${ }^{6566}$

A recent review suggested a treatment algorithm of severe irAEs ${ }^{67}$ based on histology of the affected organs and the dominant infiltrating cell types, perhaps this framework could be extended to patients who develop toxicity with pre-existing AIDs as well. Currently, there is not enough evidence to support autoantibody testing in patients without pre-existing AID before the initiation of ICIs. If this is beneficial after the development of irAEs is also unclear at present.

One major limitation of this review is that many of the studies evaluating ICI safety evaluated many AIDs in aggregate. While these findings are encouraging, the substantial heterogeneity of AID in some of these studies, which may have different etiologies, risks oversimplifying the use of ICI in these patients. However, the data do seem to suggest that these drugs can often be used safely and with encouraging responses in many tumor types. In a monitored setting where rheumatologists and oncologists can collaborate in care, a prior diagnosis of AID should not preclude potentially lifesaving or life-prolonging cancer treatment with ICI. To this end, ongoing prospective clinical studies of ICIs in patients with pre-existing autoimmune illness may illuminate the complex relationship between irAEs and pre-existing autoimmunity. A multisite NCI-sponsored prospective cohort study called, 'Nivolumab in Treating Patients with Autoimmune Disorders or Advanced, Metastatic, or Unresectable Cancer' (AIM-NIVO, NCT03816345) is aimed at addressing the safety and efficacy of ICI therapy in patients with specific pre-existing AIDs and intercurrent malignancy. This study will subdivide AIDs into distinct cohorts, making the risk of ICIs in specific AIDs easier to assess. These data are desperately needed to advance this nascent field and inform treatment guidelines for patients with a diagnosis of both AID and malignancy.

Twitter Patrick Boland @dr_boland and Sabina Sandigursky @sandigursky

Contributors SS and PB developed the concept, reviewed the literature and wrote the manuscript. JW and ACP provided invaluable editing and review of the manuscript.

Funding The authors have not declared a specific grant for this research from any funding agency in the public, commercial or not-for-profit sectors.

Competing interests JW consults for and has received less than US\$10,000 dollars per annum from Merck, Genentech, Astra Zeneca, GSK, Novartis, Nektar, Medivation, Celldex, Incyte and EMD Serono and US\$10-25,000 dollars from BMS for membership on Advisory Boards, holds equity in CytoMx, Biond and Altor, is on a scientific advisory board for Celldex, CytoMx, Incyte, Biond, Protean, CV6 and Sellas and was named on a patent from Moffitt Cancer Center on an ipilimumab biomarker and a PD-1 patent from Biodesix.

\section{Patient consent for publication Not required.}

Provenance and peer review Not commissioned; externally peer reviewed.

Open access This is an open access article distributed in accordance with the Creative Commons Attribution Non Commercial (CC BY-NC 4.0) license, which permits others to distribute, remix, adapt, build upon this work non-commercially, and license their derivative works on different terms, provided the original work is properly cited, appropriate credit is given, any changes made indicated, and the use is non-commercial. See http://creativecommons.org/licenses/by-nc/4.0/.

\section{REFERENCES}

1 Ladouceur A, Bernatsky S, Ramsey-Goldman R, et al. Managing cancer risk in patients with systemic lupus erythematous. Expert Rev Clin Immunol 2018;14:793-802.

2 Simon TA, Thompson A, Gandhi KK, et al. Incidence of malignancy in adult patients with rheumatoid arthritis: a meta-analysis. Arthritis Res Ther 2015;17:212.

3 Wang W, Macaulay W. Opioids vs Nonopioids for chronic back, hip, or knee pain. JAMA 2018;320:506-7.

4 Khan SA, Pruitt SL, Xuan L, et al. Prevalence of autoimmune disease among patients with lung cancer: implications for immunotherapy treatment options. JAMA Oncol 2016;2:1507-8.

5 Kennedy LC, Bhatia S, Thompson JA, et al. Preexisting autoimmune disease: implications for immune checkpoint inhibitor therapy in solid tumors. J Natl Compr Canc Netw 2019;17:750-7.

6 Hodi FS, O'Day SJ, McDermott DF, et al. Improved survival with ipilimumab in patients with metastatic melanoma. $N$ Engl J Med 2010;363:711-23.

7 Eggermont AMM, Chiarion-Sileni V, Grob J-J, et al. Prolonged survival in stage III melanoma with ipilimumab adjuvant therapy. $N$ Engl J Med 2016;375:1845-55.

8 Weber JS, Kähler KC, Hauschild A. Management of immune-related adverse events and kinetics of response with ipilimumab. J Clin Oncol 2012;30:2691-7.

9 Pai C-CS, Simons DM, Lu X, et al. Tumor-conditional anti-CTLA4 uncouples antitumor efficacy from immunotherapy-related toxicity. $J$ Clin Invest 2019;129:349-63.

10 Weber JS, Hodi FS, Wolchok JD, et al. Safety profile of nivolumab monotherapy: a pooled analysis of patients with advanced melanoma. J Clin Oncol 2017;35:785-92.

11 Robert C, Schachter J, Long GV, et al. Pembrolizumab versus ipilimumab in advanced melanoma. N Engl J Med 2015;372:2521-32.

12 Brahmer J, Reckamp KL, Baas P, et al. Nivolumab versus docetaxel in advanced squamous-cell non-small-cell lung cancer. $N$ Engl J Med 2015;373:123-35.

13 Topalian SL, Hodi FS, Brahmer JR, et al. Five-Year survival and correlates among patients with advanced melanoma, renal cell carcinoma, or non-small cell lung cancer treated with nivolumab. JAMA Oncol 2019. doi:10.1001/jamaoncol.2019.2187. [Epub ahead of print: 25 Jul 2019].

14 Khunger M, Rakshit S, Pasupuleti V, et al. Incidence of Pneumonitis With Use of Programmed Death 1 and Programmed Death-Ligand 1 Inhibitors in Non-Small Cell Lung Cancer: A Systematic Review and Meta-Analysis of Trials. Chest 2017;152:271-81.

15 Pillai RN, Behera M, Owonikoko TK, et al. Comparison of the toxicity profile of PD-1 versus PD-L1 inhibitors in non-small cell lung cancer: a systematic analysis of the literature. Cancer 2018;124:271-7.

16 Kelly K, Infante JR, Taylor MH, et al. Safety profile of avelumab in patients with advanced solid tumors: a pooled analysis of data from the phase 1 javelin solid tumor and phase 2 javelin Merkel 200 clinical trials. Cancer 2018;124:2010-7.

17 Martins F, Sofiya L, Sykiotis GP, et al. Adverse effects of immunecheckpoint inhibitors: epidemiology, management and surveillance. Nat Rev Clin Oncol 2019;16:563-80.

18 Wolchok JD, Chiarion-Sileni V, Gonzalez R, et al. Overall survival with combined nivolumab and ipilimumab in advanced melanoma. $N$ Engl J Med 2017;377:1345-56.

19 Motzer RJ, Tannir NM, McDermott DF, et al. Nivolumab plus ipilimumab versus sunitinib in advanced renal-cell carcinoma. $N$ Engl J Med 2018;378:1277-90.

20 Leonardi GC, Gainor JF, Altan M, et al. Safety of programmed death-1 pathway inhibitors among patients with non-small-cell lung cancer and preexisting autoimmune disorders. J Clin Oncol 2018;36:1905-12.

21 Postow MA, Chesney J, Pavlick AC, et al. Nivolumab and ipilimumab versus ipilimumab in untreated melanoma. $N$ Engl $\mathrm{J}$ Med 2015;372:2006-17. 
22 Larkin J, Chiarion-Sileni V, Gonzalez R, et al. Combined nivolumab and ipilimumab or monotherapy in untreated melanoma. N Engl J Med 2015;373:23-34.

23 Johnson DB, Balko JM, Compton ML, et al. Fulminant myocarditis with combination immune checkpoint blockade. N Engl J Med 2016;375:1749-55.

24 Zamami Y, Niimura T, Okada N, et al. Factors associated with immune checkpoint Inhibitor-Related myocarditis. JAMA Oncol 2019 doi:10.1001/jamaoncol.2019.3113. [Epub ahead of print: 22 Aug 2019].

25 Moslehi JJ, Salem J-E, Sosman JA, et al. Increased reporting of fatal immune checkpoint inhibitor-associated myocarditis. Lancet 2018;391:933

26 Bertrand F, Montfort A, Marcheteau E, et al. Tnfo blockade overcomes resistance to anti-PD-1 in experimental melanoma. Nat Commun 2017;8:2256.

27 Perez-Ruiz E, Minute L, Otano I, et al. Prophylactic TNF blockade uncouples efficacy and toxicity in dual CTLA-4 and PD-1 immunotherapy. Nature 2019;569:428-32.

28 Badran YR, Cohen JV, Brastianos PK, et al. Concurrent therapy with immune checkpoint inhibitors and TNF $\alpha$ blockade in patients with gastrointestinal immune-related adverse events. J Immunother Cancer 2019;7:226.

29 Toi Y, Sugawara S, Kawashima Y, et al. Association of immunerelated adverse events with clinical benefit in patients with advanced non-small-cell lung cancer treated with nivolumab. Oncologist 2018;23:1358-65.

30 Freeman-Keller M, Kim Y, Cronin H, et al. Nivolumab in resected and unresectable metastatic melanoma: characteristics of immunerelated adverse events and association with outcomes. Clin Cancer Res 2016;22:886-94.

31 Indini A, Di Guardo L, Cimminiello C, et al. Immune-Related adverse events correlate with improved survival in patients undergoing antiPD1 immunotherapy for metastatic melanoma. J Cancer Res Clin Oncol 2019;145:511-21.

32 Haratani K. Department of medical oncology KUFoM, OsakaSayama, Japan, Hayashi H, department of medical oncology KUFoM, Osaka-Sayama, Japan, Chiba Y, clinical research center $\mathrm{KUH}$, Osaka-Sayama, Japan, et al. Association of immune-related adverse events with nivolumab efficacy in Non-Small-Cell lung cancer. JAMA Oncology 2019;4:374-8.

33 Das S, Johnson DB. Immune-Related adverse events and anti-tumor efficacy of immune checkpoint inhibitors. J Immunother Cancer 2019;7:306

34 Johnson DB, McDonnell WJ, Gonzalez-Ericsson PI, et al. A case report of clonal EBV-like memory $\mathrm{CD} 4^{+} \mathrm{T}$ cell activation in fatal checkpoint inhibitor-induced encephalitis. Nat Med 2019;25:1243-50.

35 Suresh K, Naidoo J, Zhong Q, et al. The alveolar immune cell landscape is dysregulated in checkpoint inhibitor pneumonitis. $J$ Clin Invest 2019;130:4305-15.

36 Oh DY, Cham J, Zhang L, et al. Immune toxicities Elicted by CTLA-4 blockade in cancer patients are associated with early diversification of the T-cell repertoire. Cancer Res 2017;77:1322-30.

37 Syrigos KN, Charalambopoulos A, Pliarchopoulou K, et al. The prognostic significance of autoantibodies against dsDNA in patients with colorectal adenocarcinoma. Anticancer Res 2000;20:4351-3.

38 Blaes $\mathrm{F}$, Klotz M, Huwer $\mathrm{H}$, et al. Antineural and antinuclear autoantibodies are of prognostic relevance in non-small cell lung cancer. Ann Thorac Surg 2000;69:254-8.

39 Fernández-Madrid F, VandeVord PJ, Yang X, et al. Antinuclear antibodies as potential markers of lung cancer. Clin Cancer Res 1999:5:1393-400.

40 Bei R, Masuelli L, Palumbo C, et al. A common repertoire of autoantibodies is shared by cancer and autoimmune disease patients: inflammation in their induction and impact on tumor growth. Cancer Lett 2009;281:8-23.

41 Frisoni L, McPhie L, Colonna L, et al. Nuclear autoantigen translocation and autoantibody opsonization lead to increased dendritic cell phagocytosis and presentation of nuclear antigens: a novel pathogenic pathway for autoimmunity? J Immunol 2005;175:2692-701.

42 Toi Y, Sugawara S, Sugisaka J, et al. Profiling preexisting antibodies in patients treated with anti-PD-1 therapy for advanced non-small cell lung cancer. JAMA Oncol 2019;5:376-83.

43 Duarte JDG, Parakh S, Andrews MC, et al. Autoantibodies may predict immune-related toxicity: results from a phase I study of intralesional Bacillus Calmette-Guérin followed by ipilimumab in patients with advanced metastatic melanoma 2018.
44 de Moel EC, Rozeman EA, Kapiteijn EH, et al. Autoantibody development under treatment with Immune-Checkpoint inhibitors. Cancer Immunol Res 2019;7:6-11.

45 Gowen MF, Giles KM, Simpson D, et al. Baseline antibody profiles predict toxicity in melanoma patients treated with immune checkpoint inhibitors. J Trans/ Med 2018;16:82.

46 Good-Jacobson KL, Szumilas CG, Chen L, et al. Pd-1 regulates germinal center $B$ cell survival and the formation and affinity of longlived plasma cells. Nat Immuno/ 2010;11:535-42.

47 Nishimura H, Minato N, Nakano T, et al. Immunological studies on PD-1 deficient mice: implication of PD-1 as a negative regulator for $\mathrm{B}$ cell responses. Int Immunol 1998;10:1563-72.

48 Haas KM. Programmed cell death 1 suppresses B-1b cell expansion and long-lived IgG production in response to $T$ cell-independent type 2 antigens. J Immunol 2011;187:5183-95.

49 Stamatouli AM, Quandt Z, Perdigoto AL, et al. Collateral damage: insulin-dependent diabetes induced with checkpoint inhibitors. Diabetes 2018;67:1471-80.

50 Bingley PJ. Clinical applications of diabetes antibody testing. J Clin Endocrinol Metab 2010:95:25-33.

51 Kyi C, Carvajal RD, Wolchok JD, et al. Ipilimumab in patients with melanoma and autoimmune disease. J Immunother Cancer 2014;2:35.

52 Lee B, Wong A, Kee D, et al. The use of ipilimumab in patients with rheumatoid arthritis and metastatic melanoma. Ann Oncol 2016;27:1174-7.

53 Robert C, Thomas L, Bondarenko I, et al. Ipilimumab plus dacarbazine for previously untreated metastatic melanoma. N Engl J Med 2011;364:2517-26.

54 Hodi FS, O'Day SJ, McDermott DF, et al. Improved survival with ipilimumab in patients with metastatic melanoma. N Engl $\mathrm{J}$ Med 2010;363:711-23.

55 Johnson DB, Sullivan RJ, Ott PA, et al. Ipilimumab therapy in patients with advanced melanoma and preexisting autoimmune disorders. JAMA Oncol 2016;2:234-40.

56 Menzies AM, Johnson DB, Ramanujam S, et al. Anti-Pd-1 therapy in patients with advanced melanoma and preexisting autoimmune disorders or major toxicity with ipilimumab. Ann Oncol 2017;28:368-76.

57 Gutzmer R, Koop A, Meier F, et al. Programmed cell death protein-1 (PD-1) inhibitor therapy in patients with advanced melanoma and preexisting autoimmunity or ipilimumab-triggered autoimmunity. Eur J Cancer 2017;75:24-32.

58 Danlos F-X, Voisin A-L, Dyevre V, et al. Safety and efficacy of anti-programmed death 1 antibodies in patients with cancer and pre-existing autoimmune or inflammatory disease. Eur $J$ Cancer 2018;91:21-9.

59 Richter MD, Pinkston O, Kottschade LA, et al. Brief report: cancer immunotherapy in patients with preexisting rheumatic disease: the Mayo clinic experience. Arthritis Rheumatol 2018;70:356-60.

60 Abdel-Wahab N, Shah M, Lopez-Olivo MA, et al. Use of immune checkpoint inhibitors in the treatment of patients with cancer and preexisting autoimmune disease: a systematic review. Ann Intern Med 2018:168:121-30.

61 Keir ME, Liang SC, Guleria I, et al. Tissue expression of PD-L1 mediates peripheral T cell tolerance. J Exp Med 2006;203:883-95.

62 Tison A, Quéré G, Misery L, et al. Safety and efficacy of immune checkpoint inhibitors in patients with cancer and preexisting autoimmune disease: a nationwide, multicenter cohort study. Arthritis Rheumatol 2019;71:2100-11.

63 Abu-Sbeih H, Faleck DM, Ricciuti B, et al. Immune checkpoint inhibitor therapy in patients with preexisting inflammatory bowel disease. J Clin Oncol 2020;38:Jco1901674:576-83.

64 Efuni E, Cytryn S, Boland P, et al. Risk of toxicity after initiating immune checkpoint inhibitor treatment in patients with rheumatoid arthritis. $J$ Clin Rheumatol 2020. doi:10.1097/RHU.0000000000001314. [Epub ahead of print: 22 Jan 2020].

65 Cole S, Zibelman M, Bertino E, et al. Managing Immuno-Oncology toxicity: top 10 innovative institutional solutions. Am Soc Clin Oncol Educ Book 2019;39:96-104

66 Naidoo J, Zhang J, Lipson EJ, et al. A multidisciplinary toxicity team for cancer Immunotherapy-Related adverse events. J Natl Compr Canc Netw 2019;17:712-20.

67 Martins F, Sykiotis GP, Maillard M, et al. New therapeutic perspectives to manage refractory immune checkpoint-related toxicities. Lancet Oncol 2019;20:e54-64.

68 Kähler KC, Eigentler TK, Gesierich A, et al. Ipilimumab in metastatic melanoma patients with pre-existing autoimmune disorders. Cancer Immunol Immunother 2018;67:825-34.

69 Cortellini A, Buti S, Santini D, et al. Clinical outcomes of patients with advanced cancer and pre-existing autoimmune diseases treated with 
